\title{
Article \\ Germanium Quantum-Dot Array with Self-Aligned Electrodes for Quantum Electronic Devices
}

\author{
I-Hsiang Wang, Po-Yu Hong, Kang-Ping Peng, Horng-Chih Lin, Thomas George and Pei-Wen Li *
}

check for updates

Citation: Wang, I.-H.; Hong, P.-Y.; Peng, K.-P.; Lin, H.-C.; George, T.; Li, P.-W. Germanium Quantum-Dot Array with Self-Aligned Electrodes for Quantum Electronic Devices. Nanomaterials 2021, 11, 2743. https:// doi.org/10.3390/nano11102743

Academic Editor: Jong-Soo Lee

Received: 11 September 2021

Accepted: 13 October 2021

Published: 16 October 2021

Publisher's Note: MDPI stays neutral with regard to jurisdictional claims in published maps and institutional affiliations.

Copyright: (c) 2021 by the authors. Licensee MDPI, Basel, Switzerland. This article is an open access article distributed under the terms and conditions of the Creative Commons Attribution (CC BY) license (https:// creativecommons.org/licenses/by/ $4.0 /)$.
Institute of Electronics, National Yang Ming Chiao Tung University, Hsin Chu City 30010, Taiwan; wang.ee06g@nctu.edu.tw (I.-H.W.); allenhong1113@gmail.com (P.-Y.H.); pengkp@hotmail.com (K.-P.P.); hclin@nycu.edu.tw (H.-C.L.); tg4786125@gmail.com (T.G.)

* Correspondence: pwli@nycu.edu.tw

\begin{abstract}
Semiconductor-based quantum registers require scalable quantum-dots (QDs) to be accurately located in close proximity to and independently addressable by external electrodes. Si-based QD qubits have been realized in various lithographically-defined $\mathrm{Si} / \mathrm{SiGe}$ heterostructures and validated only for milli-Kelvin temperature operation. QD qubits have recently been explored in germanium $(\mathrm{Ge})$ materials systems that are envisaged to operate at higher temperatures, relax lithographic-fabrication requirements, and scale up to large quantum systems. We report the unique scalability and tunability of Ge spherical-shaped QDs that are controllably located, closely coupled between each another, and self-aligned with control electrodes, using a coordinated combination of lithographic patterning and self-assembled growth. The core experimental design is based on the thermal oxidation of poly-SiGe spacer islands located at each sidewall corner or included-angle location of $\mathrm{Si}_{3} \mathrm{~N}_{4} / \mathrm{Si}$-ridges with specially designed fanout structures. Multiple Ge QDs with good tunability in QD sizes and self-aligned electrodes were controllably achieved. Spherical-shaped Ge QDs are closely coupled to each other via coupling barriers of $\mathrm{Si}_{3} \mathrm{~N}_{4}$ spacer layers/c-Si that are electrically tunable via self-aligned poly-Si or polycide electrodes. Our ability to place size-tunable spherical Ge QDs at any desired location, therefore, offers a large parameter space within which to design novel quantum electronic devices.
\end{abstract}

Keywords: germanium; quantum dot; self-aligned electrode; scalability

\section{Introduction}

Since the inception of quantum computing in the early 1980, extensive research on photons [1], ion traps [2], superconducting circuits [3], and semiconductor quantum dots (QDs) [4-6] has resulted in spectacular advances in quantum-bit (qubit) technologies potentially facilitating a vast landscape of applications. While impressive achievements have been made using superconducting qubits operating at $\mathrm{mK}$ temperatures, semiconductor QD qubits have recently emerged as the subject of intensive research not only for the promise of scalability, but also for their ease of manufacturability using existing very large scale integrated circuits (VLSI) technologies [7-16]. Pioneering studies on III-V QDs have led to important proofs-of-concept for coherent control of electron-electron and electronspin interactions [4,5] Group IV semiconductors, Si and Ge, subsequently advanced these concepts to a more practical level due to their promise of relatively straightforward integration with complementary metal-oxide-semiconductor (CMOS) electronics for effective qubit control, read/write, and subsequent signal processing [8-16]. Long spin coherence times for the zero-nuclear-spin isotopes of ${ }^{28} \mathrm{Si}$ and ${ }^{74} \mathrm{Ge}$, in particular, have made both $\mathrm{Si}$ and Ge attractive as the host materials for QD-based spin qubits and affiliated single-electron transistors (SETs) exploiting their charge and spin degrees of freedom [13-15].

Semiconductor quantum computers require scalable QD qubits to be accurately located in close proximity to each other and also be independently addressable by external electrodes via tunable coupling. To date, advances in Si-based qubit technology have been 
demonstrated mostly using lithographically-defined approaches including electrostaticallyinduced QDs and physically-etched QDs based on two-dimensional electron-gas (2DEG) or hole-gas (2DHG) heterostructures [8-17] and one-dimensional nanowire structures [18,19]. Among the demonstrated electrostatically-induced QD techniques, overlapping gate architectures $[8,20]$ have offered some flexibility in forming gate-controlled QDs with electricallytunable coupling between adjacent QDs. At least $2 \mathrm{~N}+1$ control electrodes are required for defining N QDs and creating their confinement barriers. That is, $N$ plunger gates (PGs) are required to set the potential and charge occupation within the QDs in combination with $\mathrm{N}+$ 1 intervening barrier gates (BGs) to adjust inter-QD exchange interactions and QD-reservoir coupling. $\mathrm{Si} / \mathrm{Si}_{1}{ }_{\mathrm{x}} \mathrm{Ge}_{\mathrm{x}}$ double QDs (DQDs) [10-14,18], triple QDs (TQDs) [21], quadruple QDs (QQDs) [22], and octuple QDs (OQDs) [23] in linear-chain and two-dimensional arrays have been reported for qubit logic gates. But, filling charges within a specific QD among a large overlapping-gate QD array still faces difficult technical challenges such as effective elimination/reduction of qubit cross-talk and quantum-state leakage. The overlappinggate architecture results in individual gate voltages not only modulating the specific QD potentials or inter-QD coupling that they are designed to control, but also influencing parameters of other, unintentionally-addressed QDs through capacitive cross-talk [24,25]. Also, gate-induced disk- or circular-shaped QDs based on 2DEG/2DHG heterostructures, which have diameters much larger than their thickness, can result in highly anisotropic potential confinement $[17,26]$. The transverse potential in these structures, in particular, exhibits cylindrical symmetry with a soft-wall profile, resulting in weak confinement and hyperfine energy-level splitting. These effects have resulted in the operation of $\mathrm{Si} / \mathrm{SiGe}$ qubits only being validated at very low temperatures $(\leq 2 \mathrm{~K})[8,16]$. Although physically etched QD approaches do indeed increase the freedom for implementing QD devices with diverse spatial orientations and locations [27], a major challenge that remains is the formation of electrical contacts to specific QDs even using the most advanced lithographic techniques available. Besides, hard-wall confined QDs can lead to fixed tunneling rates and fixed exchange interactions between QDs [28], making it extremely difficult to reliably measure very small output current/voltage signals.

For the proper functioning of QD qubits with high fidelity, it is vital to fabricate reliable and scalable QDs with a high degree of control over the QD size, shape, crystallinity, strain, and inter-QD spacing. In particular, the physical dimensions of QDs and their coupling barriers must be sufficiently small at nanometer-scale levels [17]. This last requirement has proven challenging from a fabrication perspective. Controllably producing ultrasmall Si QDs, since this is dictated by the small Bohr radius of $4.9 \mathrm{~nm}$ in $\mathrm{Si}$, is difficult using lithographic techniques alone. In contrast, a larger Bohr radius of 24.9 $\mathrm{nm}$ in Ge enables easier modification of Ge QD-based device structures, imposing far less stringent demands on lithographic control as compared to Si QD fabrication. Also the co-existence of long electron-spin relaxation times with strong spin-orbit coupling in Ge permits electrically-driven manipulation for fast operation [29]. Encouragingly, the proof-of-principle Ge qubit devices have been experimentally demonstrated using Ge/SiGe planar heterostructures [15,30], Ge hut wires [31], and Ge/Si core/shell nanowires [32], respectively. Progresses in the optimization of Ge hole-based qubit devices based on these material platforms have excited important achievements in terms of large g-factors and spin-orbit interaction energies [33]. Each of these platforms offers specific advantages but also poses challenges, which have been comprehensively elaborated and reviewed in [33]. Thus far, operation of two- and 4-qubit logic gates at $\mathrm{mK}$ temperatures has been demonstrated using large Ge QDs (5-70 $\mathrm{nm}$ in height and $100 \mathrm{~nm}$ in planar dimensions) with an inter-QD pitch of 150-200 $\mathrm{nm}$ based on gate-defined SiGe/Ge/SiGe quantum-wells on $\mathrm{Si}$ substrates $[15,30,34]$.

We have reported a CMOS-compatible fabrication approach for the controllable growth of spherical-shaped $\mathrm{Ge} \mathrm{QDs} / \mathrm{SiO}_{2}$ shells within Si-containing layers $\left(\mathrm{SiO}_{2}, \mathrm{Si}_{3} \mathrm{~N}_{4}\right.$, and $\mathrm{Si}$ ) in a self-organized manner [35-38]. Using a coordinated combination of lithographic patterning and self-assembled growth, size-tunable Ge QDs were controllably 
positioned by successfully exploiting the many peculiar and symbiotic interactions of $\mathrm{Si}$, $\mathrm{Ge}$, and O interstitials [39-41]. Our Ge QDs were created using the selective oxidation of poly-Si $i_{1} \mathrm{Ge}_{\mathrm{x}}$ lithographically-patterned structures with $\mathrm{Si}_{3} \mathrm{~N}_{4}$ in proximity. We have exploited the multi-dimensional parameter spaces of process conditions to grow Ge QDs with a high degree of controllability in the size, morphological shape, chemical purity, crystallinity, and spatial locations [39-47]. We have also proven the feasibility of paired DQDs embedded within $\mathrm{SiO}_{2} / \mathrm{Si}_{3} \mathrm{~N}_{4}$ matrices at each sidewall edge of lithographicallypatterned Si ridges using spacer technology and thermal oxidation of poly-SiGe $[37,38]$. The inherent structural simplicity of our self-organized $\mathrm{Ge} \mathrm{QD} / \mathrm{SiO}_{2}$ shell heterostructures perfectly enables the experimental realization of Ge-QD single-hole transistors (SHTs) [42-44]. Well-resolved tunneling current spectroscopy and superior charge stabilities measured at $\mathrm{T}=77-150 \mathrm{~K}[43,44]$, suggests that our Ge-QD SHTs are effective charge sensors.

In this paper, we advance the self-aligned fabrication of ordered arrays of Ge QDs closely coupled with each other via $\mathrm{Si}_{3} \mathrm{~N}_{4}$ spacer layers/c-Si ridges that serve as inter-QD coupling barriers. The core experimental design is based on the thermal oxidation of poly$\mathrm{SiGe}$ spacer islands located at each included-angle location of specially designed $\mathrm{Si}_{3} \mathrm{~N}_{4} / \mathrm{Si}-$ ridges (Figure 1). By tailoring the specially designed fanout structures, Ge multiple QDs with good tunability in QD sizes were controllably generated at each included-angle location of Asterisk-shaped $\mathrm{Si}_{3} \mathrm{~N}_{4} / \mathrm{Si}$ ridges.

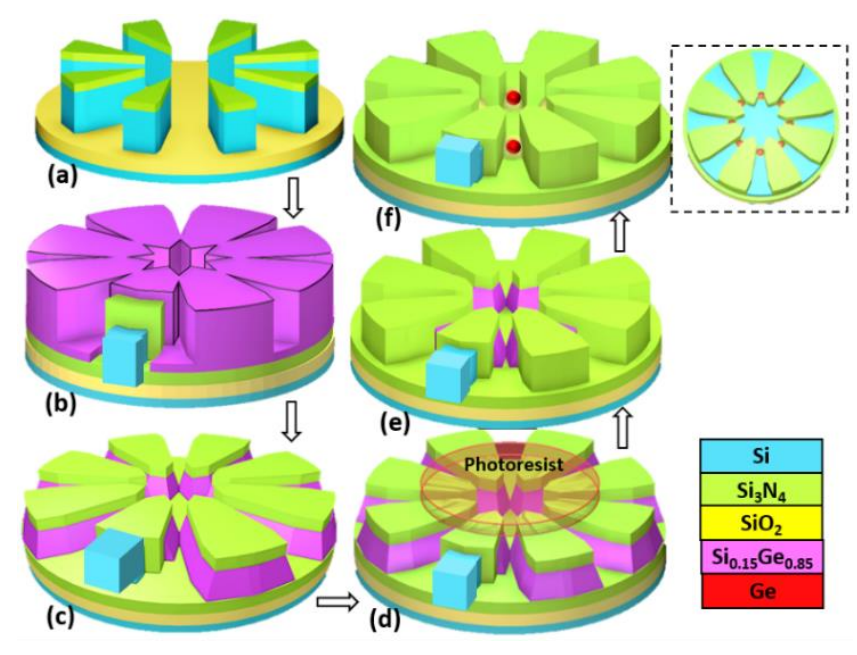

Figure 1. Process flow diagrams showing the fabrication of multiple QDs embedded within $\mathrm{SiO}_{2} / \mathrm{Si}_{3} \mathrm{~N}_{4}$ matrices via the thermal oxidation of $\mathrm{SiGe}$ spacer islands at designated included-angle locations of $\mathrm{Si}_{3} \mathrm{~N}_{4}$ /c-Si ridges. (a) Lithographically patterned $\mathrm{Si}_{3} \mathrm{~N}_{4} / \mathrm{c}$-Si fanout ridges on top of an SOI substrate. (b) Next, sequential deposition of $\mathrm{Si}_{3} \mathrm{~N}_{4}$ and poly-Si $\mathrm{i}_{0.85} \mathrm{Ge}_{0.15}$ layers conformally encapsulates the $\mathrm{Si}_{3} \mathrm{~N}_{4}$ /c-Si ridges. (c) Symmetrical spacer stripes of poly-Si ${ }_{0.85} \mathrm{Ge}_{0.15}$ are subsequently fabricated at each sidewall of the $\mathrm{Si}_{3} \mathrm{~N}_{4} / \mathrm{c}$-Si ridges by a direct etch back process. (d) Lithographic-patterning shadowing the central regions of the designed fanout ridges in combination with (e) etching processes are conducted to define the lengths of the poly- $\mathrm{Si}_{0.85} \mathrm{Ge}_{0.15}$ spacer islands. (f) Next, spherical Ge QDs are formed at each included-angle location of the nano-patterned $\mathrm{Si}_{3} \mathrm{~N}_{4} / \mathrm{c}-\mathrm{Si}$ ridges sidewall by thermal oxidation. The inset is the plan-view sketch showing the simultaneous formation of multiple Ge QDs at each included-angle locations of $\mathrm{Si}_{3} \mathrm{~N}_{4}$ /c-Si ridges by design.

\section{Experimental Methods and Procedures}

The experimental procedure for the fabrication of self-organized Ge multiple QDs with coupling barriers of $\mathrm{Si}_{3} \mathrm{~N}_{4}$ /c-Si ridges and self-aligned $\mathrm{Si}$ electrodes (BGs, PGs, and reservoirs) is described in Figures 1 and 2, respectively. Starting with a silicon-on-insulator (SOI) substrate comprising a $100 \mathrm{~nm}$-thick single-crystalline $\mathrm{Si}$ (c-Si) layer and a $400 \mathrm{~nm}$ thick buried $\mathrm{SiO}_{2}$ layer on top of $\mathrm{Si}$ substrate, a $25 \mathrm{~nm}$-thick $\mathrm{Si}_{3} \mathrm{~N}_{4}$ layer was deposited 
using low-pressure chemical vapor deposition (LPCVD) as the hard-mask layer for the subsequent processes of plasma etching and thermal oxidation. Specially designed Asteriskshaped Si ridges were subsequently produced using a combination of electron-beam lithographic (EBL) patterning and $\mathrm{SF}_{6} / \mathrm{C}_{4} \mathrm{~F}_{8}$ plasma etching (Figure 1a). Next, bi-layers of $10 \mathrm{~nm}$-thick $\mathrm{Si}_{3} \mathrm{~N}_{4}$ and $25-30 \mathrm{~nm}$-thick poly-Si ${ }_{0.85} \mathrm{Ge}_{0.15}$ were sequentially deposited using LPCVD (Figure 1b) for conformal encapsulation over the Si ridges. Following a direct etch-back process using $\mathrm{SF}_{6} / \mathrm{C}_{4} \mathrm{~F}_{8}$ plasma (Figure 1c), spacer stripes of poly-Si $\mathrm{Si}_{0.85} \mathrm{Ge}_{0.15}$ with width/height of 20-30/10-30 nm were symmetrically produced at each sidewall of the $\mathrm{Si}_{3} \mathrm{~N}_{4} / \mathrm{c}$-Si ridges by adjusting the etch-back process time. A second EBL (Figure 1d) in combination with $\mathrm{SF}_{6} / \mathrm{C}_{4} \mathrm{~F}_{8}$ plasma etching (Figure 1e) was conducted for shadowing the central regions of the Asterisk-shaped $\mathrm{Si}_{3} \mathrm{~N}_{4} / \mathrm{Si}$ ridges, respectively. In this way we defined the lengths of the poly-Si ${ }_{0.85} \mathrm{Ge}_{0.15}$ spacer islands at each included angle location of the $\mathrm{Si}_{3} \mathrm{~N}_{4} / \mathrm{Si}$ ridges (Figure 1e). Subsequently, thermal oxidation at $900{ }^{\circ} \mathrm{C}$ for $25-40 \mathrm{~min}$ in an $\mathrm{H}_{2} \mathrm{O}$ ambient was performed to convert these poly- $\mathrm{Si}_{0.85} \mathrm{Ge}_{0.15}$ spacer islands to $\mathrm{Ge}$ QDs with cladding oxide layers (Figure 1f) at designated locations by the ridges.

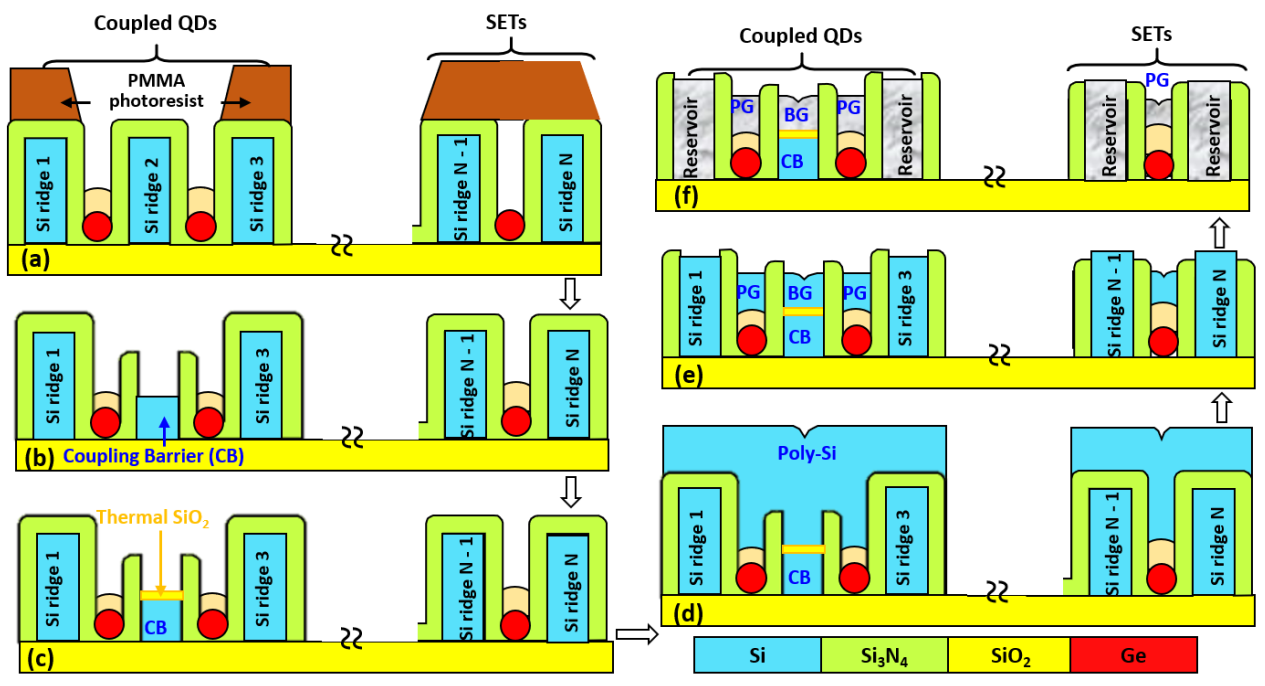

Figure 2. Process flow diagrams showing the fabrication of self-aligned PGs, BGs, and source/drain reservoirs. (a) EBL opening of the selected c-Si ridges (ridge \#2) that would serve as CBs; (b) the removal of the top $\mathrm{Si}_{3} \mathrm{~N}_{4}$ and the top portion of the selected c-Si ridges (ridge \#2) using $\mathrm{CHF}_{3}$ plasma and $\mathrm{SF}_{6} / \mathrm{C}_{4} \mathrm{~F}_{8}$ plasma, respectively, forming $\mathrm{CBs}$; (c) the growth of a thin thermal $\mathrm{SiO}_{2}$ over the CBs; (d) deposition of poly-Si overlayers; (e) direct etch back of poly-Si overlayers and top $\mathrm{Si}_{3} \mathrm{~N}_{4}$ over the outmost c-Si ridges (for instance, ridges \#1, \#3, \#N - 1, \#N), forming PGs over the Ge QDs and BGs over $\mathrm{CBs}$ via $\mathrm{SiO}_{2}$ layers; (f) silicidation of the outmost c-Si ridges, PGs, and BGs, forming polycide reservoirs, $\mathrm{PG}$, and $\mathrm{BG}$, respectively.

Following the formation of cladding oxide/Ge QDs at the included-angle locations of the fanout-ridge structures, EBL process opened the selected regions of Ge QDs and coupling barriers (CBs) of $\mathrm{Si}_{3} \mathrm{~N}_{4}$ spacers/c-Si ridges (that is, shadowing the outmost c-Si ridges with photoresists) as shown in Figure 2a. Next, the top $\mathrm{Si}_{3} \mathrm{~N}_{4}$ and the top portion ( $\sim 50 \mathrm{~nm}$-thick) of c-Si ridges (Figure $2 \mathrm{~b}$ ) were sequentially removed using $\mathrm{CHF}_{3}$ plasma and $\mathrm{SF}_{6} / \mathrm{C}_{4} \mathrm{~F}_{8}$ plasma, respectively. A subsequent thermal oxidation process grew a $5 \mathrm{~nm}$ thick $\mathrm{SiO}_{2}$ layer on top of the selected c-Si ridges (Figure 2c). Next, combined processes of deposition (Figure 2d) and direct etch-back (Figure 2e) of $100 \mathrm{~nm}$-thick poly-Si layers simultaneously form plunger gates (PGs) on top of the capping $\mathrm{SiO}_{2} / \mathrm{Ge}$ QDs and barrier gates (BGs) over the $5 \mathrm{~nm}$-thick $\mathrm{SiO}_{2} / \mathrm{c}$-Si ridges in a self-aligned approach. Finally, the poly-Si plunger gates, barrier gates, and the outmost c-Si ridges (serving as reservoirs) could be converted to metallic electrodes of NiSi by using the self-aligned silicidation processes (Figure $2 \mathrm{f}$ ). 
In this work, the critical lithographic patterning of $\mathrm{Si}$ ridges and SiGe spacer islands was conducted using Raith VOYAGER electron-beam lithography system (Raith $\mathrm{GmbH}$, Dortmund, Germany) and Oxford DSiE plasma etcher (Oxford Instruments plc, Abingdon, UK). Thin specimens for scanning transmission electron microscopy (STEM) observation were prepared by ion-beam milling in a dual-beam (focused ion beam and electron beam) TESCAN GAIA3 (TESCAN, Brno, Czech Republic) using in-situ liftout techniques in order to reduce carbon contamination levels during sample preparation. Energy dispersive x-ray spectroscopy (EDS) analyses were carried out in a FEI Titan $\mathrm{G}^{2}$ 80-200 ChemiSTEM (FEI Technologies Inc., Salem, OR, USA), equipped with a Cs probe corrector in combination with an in-column Super-X EDS (Bruker Corporation, Billerica, MA, USA) with four windowless silicon-drift detectors $\left(4 \times 30 \mathrm{~mm}^{2}\right)$ and operated at $200 \mathrm{kV}$, leading to a spatial resolution of $7 \AA$. All STEM imaging and EDS analyses were performed by using a high-angle annular dark-field (HAADF) detector (E.A. Fischione Instruments, Inc., Export, PA, USA) with convergence semi-angles of $8.24 \mathrm{mrad}$ for the inner acceptance angle and $\sim 143.6 \mathrm{mrad}$ for the outer acceptance angle at spot size 9. The characteristic $\mathrm{X}$-ray fluorescence energy lines for Germanium, Silicon, Nitrogen, and Oxygen are Ge-K $\alpha$ : $9.871 \mathrm{keV}, \mathrm{Si}-\mathrm{K} \alpha$ : $1.74 \mathrm{keV}, \mathrm{N}-\mathrm{K} \alpha$ : $0.392 \mathrm{keV}$, and O-K $\alpha$ : $0.525 \mathrm{keV}$, respectively. SEM examinations were conducted using a Hitachi S-4700I field-emission scanning-electron microscope (Hitachi High-Technologies Corp., Tokyo, Japan) at an acceleration voltage of $15 \mathrm{kV}$ with a resolution of $1.5 \mathrm{~nm}$. Synchrotron X-ray diffraction (XRD) measurement was performed in the BL07 beamlines of National Synchrotron Radiation Research Center (NSRRC), Hsinchu, Taiwan. Incident X-ray (wavelength $0.6888 \AA, 18 \mathrm{keV}$ ) was generated from a superconducting undulator and, consequently, $\mathrm{X}$-ray with ultra-high flux could be obtained. When we precisely controlled two angles for single crystal diffractions in the double crystal monochromator, energy resolution of $X$-ray achieved $1.5 \times 10^{-4} \Delta \mathrm{E} / \mathrm{E}$ ). An imaging plate detector (Mar345, made by marXperts $\mathrm{GmbH}$, Norderstedt, Germany) was used to collect Laue rings, and $\mathrm{a} \mathrm{CeO}_{2}$ powder standard was used to calibrate incident $\mathrm{X}$ ray energy, sample-to-detector distance, and title/rotation of a detector. Finally, diffraction patterns were obtained as integrating Laue ring by GSAS II package.

Temperature-dependent current-voltage (I-V) measurements were conducted in a Lakeshore TTP-6 liquid-nitrogen cooled vacuum-sealed probe station (Lake Shore Cryotronics, Inc., Westerville, OH, USA) using the semiconductor device analyzer Agilent B1500A equipped with B1517A high-resolution source monitor unit/atto sense and switch unit (Keysight Technologies, Santa Rosa, CA, USA), improving the low-current measurement resolution to femtoampere range. Kevin triaxial cables were used to connect the B1500A to wafer probers for these cables producing less electrical noise, leakage, and electromotive force than doing standard triaxial cables. The set-up parameters of B1500A for the current characterization is summarized as follows: hold time: $1 \mathrm{~s}$, delay time: $10 \mathrm{~ms}$, and integration time: $0.6 \mathrm{~s}$, providing a null current of $<1 \mathrm{fA}$ at $77 \mathrm{~K}$. The differential conductance, $G_{\mathrm{D}} \equiv \partial I_{\mathrm{D}} / \partial V_{\mathrm{D}}$, was obtained by numerical smoothing measured $I_{\mathrm{D}}-V_{\mathrm{D}}$ data using a simplified least squares procedure and then making differentiation.

\section{Results}

\subsection{Formation of Self-Assembled, Closely-Coupled Ge QDs Arrays}

Our fanout fabrication process promises to ultimately achieve the controllability necessary for simultaneously forming closely-coupled multiple Ge QDs (Figure 1) with self-aligned barrier gates and plunger gates as shown in Figure 2 via adjustable coupling barriers of $\mathrm{Si}_{3} \mathrm{~N}_{4} / \mathrm{c}-\mathrm{Si}$ ridges and capping $\mathrm{SiO}_{2}$, respectively. These unique heterostructures were obtained by the thermal oxidation of poly-SiGe "spacer islands" located at each included-angle location of the specially designed, fanout-shaped Si ridges. Figure 3 shows the plan-view SEM/STEM micrographs of the key process steps for the fabrication of closely-coupled, octuple Ge QDs with diameters of $15 \mathrm{~nm}$ at each included-angle location of the c-Si fanout ridges. 

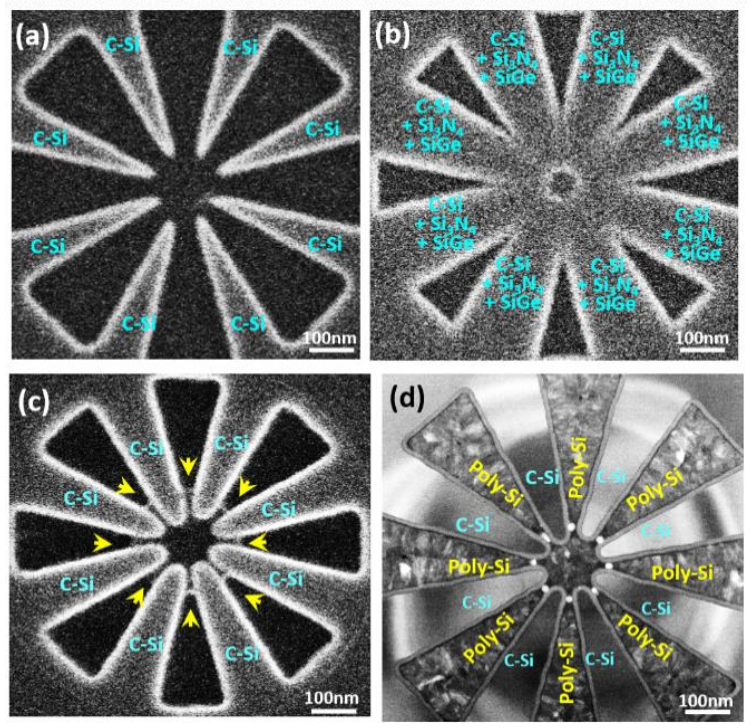

Figure 3. Plan-view SEM/TEM micrographs showing key process steps for the fabrication of closelycoupled Ge octuple QDs located at the included-angle locations of c-Si ridges with specially designed, asterisk-shaped fanout structures. SEM/TEM observations of (a) Lithographically patterned c-Si fanout ridges formed on top of SOI substrates. (b) Sequential deposition of $\mathrm{Si}_{3} \mathrm{~N}_{4}$ and poly- $\mathrm{Si}_{0.85} \mathrm{Ge}_{0.15}$ layers conformally encapsulating the c-Si ridges. (c) Symmetrical spacer layers of poly- $\mathrm{Si}_{0.85} \mathrm{Ge}_{0.15}$ fabricated at each sidewall of the $\mathrm{Si}_{3} \mathrm{~N}_{4}$ / poly-Si ridges by a direct etch back process using $\mathrm{SF}_{6} / \mathrm{C}_{4} \mathrm{~F}_{8}$ plasma. Poly-Si $\mathrm{S}_{0.85} \mathrm{Ge}_{0.15}$ spacer islands (highlighted by yellow arrows) were produced at the central regions by using lithographic-patterning and plasma-etching processes. (d) Formation of closely-coupled Ge QDs with diameter of $15 \mathrm{~nm}$ at each included-angle location of the c-Si fanout ridges following thermal oxidation. Poly-Si layers were then deposited forming plunger gates and barrier gates.

\subsection{Arrays of Ge QDs with Scalable Numbers and Tunable Diameters}

The overall number of Ge QDs in the configuration is essentially determined by the fanout number of the c-Si ridges via positioning a single Ge QD at each included-angle location. Figure 4 shows Ge OQDs configurations created by using Asterisk-shaped Si ridge geometry with eight fanouts. Process-controlled tunability of the Ge QD diameter is achieved by adjusting the overall Ge content of the poly- $\mathrm{Si}_{0.85} \mathrm{Ge}_{0.15}$ spacer island. The width and height are varied by controlling the process times for deposition and etch back, respectively, of the poly-SiGe spacer layers. Finally, the exposure dose of EBL for defining the poly-SiGe spacer islands determines their length and hence the overall Ge content. It is clearly seen from the plan-view STEM micrographs in Figure 4 that Ge OQDs with diameters of 30,15, and $8 \mathrm{~nm}$, respectively, appear at each included-angle location of the $\mathrm{Si}_{3} \mathrm{~N}_{4} / \mathrm{Si}$ ridges following thermal oxidation (at $900{ }^{\circ} \mathrm{C}$ for $25 \mathrm{~min}$ ) of poly-Si $\mathrm{i}_{0.85} \mathrm{Ge}_{0.15}$ islands with widths/heights/lengths of $30 / 45 / 60 \mathrm{~nm}, 25 / 40 / 40 \mathrm{~nm}$, and 20/20/30 nm.
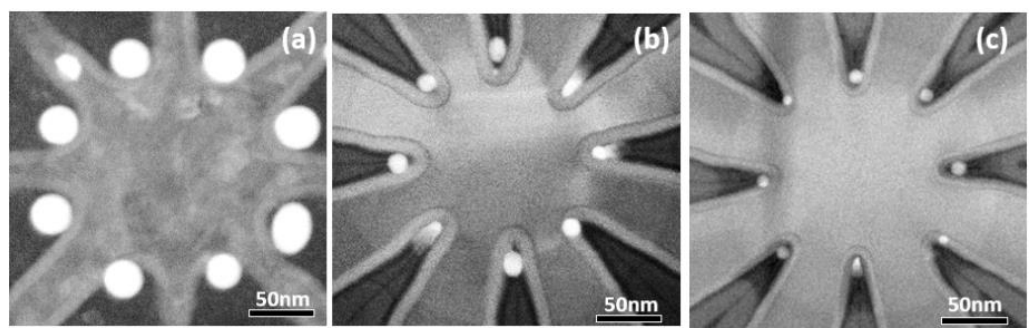

Figure 4. Plan-view STEM observations of (a) $30 \mathrm{~nm}$; (b) $15 \mathrm{~nm}$; and (c) $8 \mathrm{~nm}$ Ge octuple QDs fabricated at the sidewall corner of each included angle location for the $\mathrm{Si}_{3} \mathrm{~N}_{4}$ /asterisk-shaped c-Si ridges showing exquisite control of the number and sizes of the QDs. 


\subsection{Arrays of Ge QDs with Self-Aligned Electrodes}

The engineering advantages of our Ge QD fabrication approaches not only include process-controlled placement of size-tunable Ge QDs at designated locations, but also offer a feasible integration scheme for forming self-aligned electrodes. That is, the potentials of Ge QDs and inter-QD coupling barriers of $\mathrm{Si}_{3} \mathrm{~N}_{4} / \mathrm{c}$-Si ridges are electrically adjustable by controlling the poly-Si (or polycide) plunger-gates and barrier-gates, respectively, through thermally-grown $\mathrm{SiO}_{2}$ layers. The EDS maps of elemental $\mathrm{Si}, \mathrm{Ge}$, nitrogen $(\mathrm{N})$, and oxygen (O) micrographs in Figure 5 show that the inter-QD spacings of $30-50 \mathrm{~nm}$ are essentially determined by the widths of lithographically-patterned c-Si ridges in combination with the sidewall thicknesses of the $\mathrm{Si}_{3} \mathrm{~N}_{4}$ overlayers. That is, the $\mathrm{Si}_{3} \mathrm{~N}_{4}$ spacer layers and c-Si ridges directly define inter-QD coupling barriers (Figure $5 \mathrm{a}, \mathrm{b}$ ). Concurrent with the formation of Ge QDs, their cladding layers of $\mathrm{SiO}_{2}$ were also generated from the selective oxidation of the $\mathrm{Si}$ content of poly-SiGe spacer islands (Figure 5c).
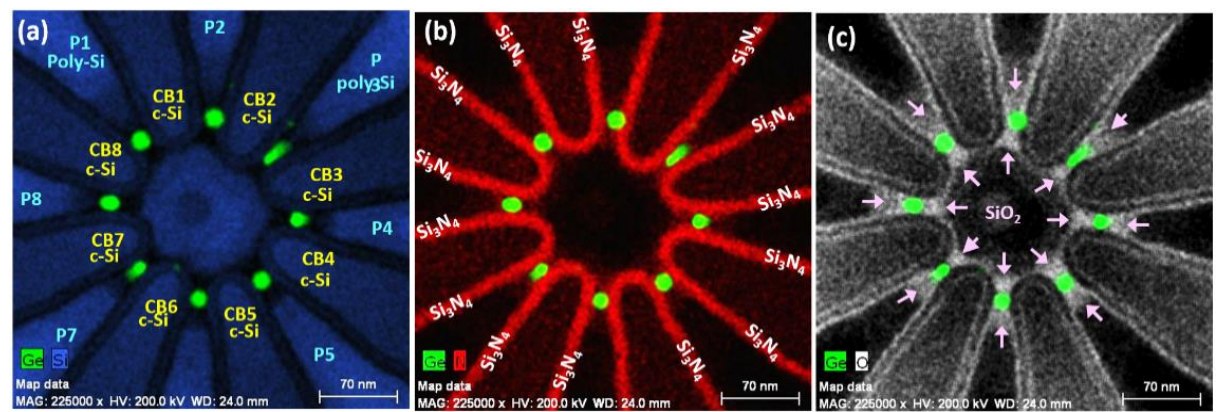

Figure 5. Plan-view EDS maps of elemental (a) silicon (Si-blue), germanium (Ge-green); (b) nitrogen (N-red), Ge; and (c) oxygen (O-white), Ge of octuple Ge QDs fabricated at each included-angle location of asterisk-shaped $\mathrm{Si}$ ridges with $\mathrm{Si}_{3} \mathrm{~N}_{4}$ overlayers.

Following the formation of Ge QDs and their cladding layers of thermally-grown $\mathrm{SiO}_{2}$, combined processes of EBL, etch-back $\left(\mathrm{Si}_{3} \mathrm{~N}_{4}\right.$ and c-Si), and thermal oxidation were sequentially conducted on the selected c-Si ridges that would serve as coupling barriers (Figure 2a,b). Subsequent deposition and etch back processes of poly-Si layers produce selfaligned poly-Si barrier gates on top of $\mathrm{SiO}_{2}$ / coupling barriers of c-Si ridges and self-aligned poly-Si plunger gates over the cladding $\mathrm{SiO}_{2} / \mathrm{Ge}$ QDs. Poly-Si barrier gates electrically adjust effective barrier width of the spacer $\mathrm{Si}_{3} \mathrm{~N}_{4}$ layers and barrier height of c-Si ridges and thereby modulate inter-QD charge-charge exchange interactions.

\section{Discussion}

Vital requirements on semiconductor QDs for functional quantum electronic devices include (1) the control over crystallinity and crystal orientations of QDs, (2) the adjustability of the QD sizes and morphological shapes with controllable positions by design, (3) good interface properties of QDs/confinement barriers, and (4) strain engineering in the QDs for valley splitting.

Our previous reports have already conducted extensive STEM-EDS and electron energy loss spectroscopy (EELS) line scan/map examinations, confirming the high chemical purity of Ge QDs (no alloyed Si or Oxygen present within the QD) [45,46]. Clear lattice fringes observed in high-resolution TEM micrographs and sharp diffraction spots observed in the selected area electron diffraction (SAED) patterns are testament to the good crystallinity of our Ge QDs [43-46]. Raman spectroscopy [46,47] and photoluminescence (PL) $[47,48]$ measurements also confirm the high degree of crystallinity within the Ge QDs in terms of sharp Raman phonon lines and temperature-insensitive PL peaks, respectively.

Our systematic Raman measurements in combination with TEM/SAED examinations also reveal an important observation that the local environments of $\mathrm{SiO}_{2}$ and $\mathrm{Si}_{3} \mathrm{~N}_{4}$ have a significant influence on the sign of the strain, tensile or compressive, which is imposed on 
the Ge QDs [46,47] That is, compressive and tensile strains can be generated in our Ge QDs depending on whether the Ge QD is embedded within $\mathrm{Si}_{3} \mathrm{~N}_{4}$ or $\mathrm{SiO}_{2}$ layers. Measured Grüneisen parameters from temperature-dependent Raman frequencies suggest significant anharmonicity for small Ge QDs with possible distortions of the diamond cubic lattice, which have been confirmed by their lattice spacings through the transmission electron diffraction patterns. We have also observed that quantum phonon confinement effect sets in when the Ge QD size is smaller than $40 \mathrm{~nm}$ [47-49]. Therefore, the valley degeneracy in our Ge QDs could be split by tailoring the local environmental materials of $\mathrm{SiO}_{2}$ or $\mathrm{Si}_{3} \mathrm{~N}_{4}$ in combination with adjusting the QD sizes by design.

From device fabrication perspectives, making source/drain reservoirs and creating tunable tunnel barriers/coupling barriers to specific, small self-assembled QDs are very challenging, in general, requiring very precise overlay alignment by means of advanced lithography. In this work, we advance the fabrication of ordered arrays of Ge QDs with self-organized tunnel barriers/coupling barriers and self-aligned electrodes. The appeals of our proposed Ge QD approach lie in the engineering advantages of controllably positioning size-tunable spherical Ge QDs with a high degree of crystallinity at desired spatial locations and thereby offering a large parameter space within which to design novel quantum electronic devices.

\subsection{Self-Organized, Crystalline $\mathrm{Ge} \mathrm{QD} / \mathrm{SiO}_{2}$-Shell with $\mathrm{Si}_{3} \mathrm{~N}_{4} / \mathrm{c}$-Si Coupling-Barrier Layers}

Our self-organized Ge QD arrays with tunable QD sizes, scalable numbers of QDs, and their controllable placement at designated locations were constructed on speciallydesigned Si-ridge structures encapsulated with conformal overlayers of $\mathrm{Si}_{3} \mathrm{~N}_{4}$. The $\mathrm{Si}_{3} \mathrm{~N}_{4}$ overlayers are pivotal for shaping and positioning the Ge QDs. It is also important to note that the $\mathrm{Si}_{3} \mathrm{~N}_{4}$ spacer layers together with the c-Si ridges directly define coupling barriers between adjacent QDs.

The fabrication process for generating our self-organized $\mathrm{Ge} \mathrm{QD} / \mathrm{SiO}_{2}$ shell within $\mathrm{Si}_{3} \mathrm{~N}_{4} / \mathrm{Si}$ layers is briefly described as follows. Thermal oxidation $\left(850-900{ }^{\circ} \mathrm{C}\right)$ of $\mathrm{Si}_{1-x} \mathrm{Ge}_{x}$ results in the preferential oxidation of its $\mathrm{Si}$ content, converting it to $\mathrm{SiO}_{2}$, due to the large difference in the heats of formation of $\mathrm{SiO}_{2}(-200 \mathrm{kcal} / \mathrm{mol})$ and $\mathrm{GeO}_{2}(-130 \mathrm{kcal} / \mathrm{mol})$ [50]. The resultant host matrices of $\mathrm{SiO}_{2}$ therefore contain a combination of pure Ge nanocrystals and residual Ge interstitials. Among our first interesting and counter-intuitive findings was the fact that the Ge nanocrystals and their associated Ge interstitial clouds catalyze the local decomposition and oxidation of the proximal $\mathrm{Si}_{3} \mathrm{~N}_{4}$ layer $[40,51]$. This decomposition process releases $\mathrm{Si}$ interstitials [39-41] that in turn, promote the Ostwald ripening and migration of the Ge nanocrystals through their surrounding $\mathrm{SiO}_{2}$ matrix in the direction of the $\mathrm{Si}$ interstitial concentration gradient towards the $\mathrm{Si}_{3} \mathrm{~N}_{4}$ layer (Figure 6a). Concurrent with their migration, the Ge nanocrystals grow in size by Ostwald Ripening culminating in complete coalescence, ultimately resulting in the formation of spherical Ge QDs embedded within $\mathrm{Si}_{3} \mathrm{~N}_{4}$ layers (Figure $6 \mathrm{~b}, \mathrm{c}$ ) with a high degree of crystallinity (Figure $6 \mathrm{~d}$ ).
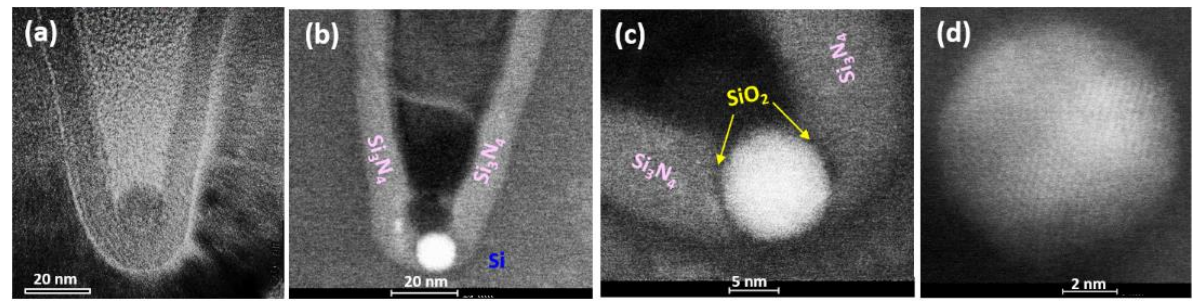

Figure 6. Plan-view TEM/STEM observations of the process evolution of Ge QDs formed at the included-angle locations of the asterisk-shaped $\mathrm{Si}_{3} \mathrm{~N}_{4} / \mathrm{Si}$ ridges undergoing thermal oxidation at $900{ }^{\circ} \mathrm{C}$ for (a) $30 \mathrm{~min}$ and (b) $40 \mathrm{~min}$. High-resolution STEM observation of (c) Ge QD with a conformal $\mathrm{SiO}_{2}$ shell penetrating the spacer layer of $\mathrm{Si}_{3} \mathrm{~N}_{4}$. Clear lattice fringes shown in (d) highresolution STEM micrograph are testament to the good crystallinity of the Ge QDs embedded within the $\mathrm{Si}_{3} \mathrm{~N}_{4}$ layers. 
The unique penetration of Ge QDs through the surrounding $\mathrm{SiO}_{2}$ matrix (Figure 6a) and proximal $\mathrm{Si}_{3} \mathrm{~N}_{4}$ layers (Figure $6 \mathrm{~b}$ ) is activated by dynamic $\mathrm{SiO}_{2}$ destruction-construction mechanisms near the QD surface [39-41]. As the Ge QD ultimately penetrates the entire $\mathrm{Si}_{3} \mathrm{~N}_{4}$ layer, a thin conformal $\mathrm{SiO}_{2}$ shell is formed separating the Ge QD and the surrounding $\mathrm{Si}_{3} \mathrm{~N}_{4}$ (Figure $6 \mathrm{c}$ ). The $\mathrm{SiO}_{2}$-shell thickness of 1-2 $\mathrm{nm}$ between the penetrating Ge QD and the $\mathrm{Si}_{3} \mathrm{~N}_{4}$ is essentially determined by a dynamic equilibrium that exists between the local concentrations of $\mathrm{O}$ interstitials near the $\mathrm{Ge} Q \mathrm{QD} / \mathrm{Si}_{3} \mathrm{~N}_{4}$ interfaces supplied by the external oxygen ambient, and combined with the concentration of Si interstitials released from the locally decomposing $\mathrm{Si}_{3} \mathrm{~N}_{4}$ layer $[40,41]$.

\subsection{Ge QD Mediated Densification of Proximal $\mathrm{Si}_{3} \mathrm{~N}_{4}$ Barriers}

The next interesting finding was that the penetrating Ge QDs also remarkably mediate the local densification of the nominally amorphous $\mathrm{Si}_{3} \mathrm{~N}_{4}$ spacer layers (Figure 7a) via a phase transition from amorphous to the nanocrystalline state, as evidenced by clear diffraction spots in the SAED patterns (Figure $7 \mathrm{~b}$ ) and sharp peaks in the XRD spectra (Figure 7c). The observed peaks at $2 \theta=29.12,48.37$, and $57.31^{\circ}$ correspond to the crystal planes of $(201),(3-12)$ or $(4-10)$, and $(4-22)$, respectively, of crystalline $\mathrm{Si}_{3} \mathrm{~N}_{4}$ in the $\alpha$-phase state of a trigonal crystal structure. The derived classification of crystal planes from the XRD spectra and the corresponding diffraction spots identified within the SAED are in good agreement. This densification of $\mathrm{Si}_{3} \mathrm{~N}_{4}$ also leads to the reduction in the concentration of hydrogen induced traps and thereby a significant improvement in the trap-assisted tunneling or hopping $[49,52]$. Low interface trap density (Dit) of $\sim 2-3 \times 10^{11} \mathrm{~cm}^{2} \mathrm{eV}^{-1}$ was measured on the Ge QD/ $\mathrm{Si}_{3} \mathrm{~N}_{4}$ structures [53], and estimated number of interface traps for a $10 \mathrm{~nm} \mathrm{Ge}$ QDs $/ \mathrm{Si}_{3} \mathrm{~N}_{4}$ structure is approximate unity.
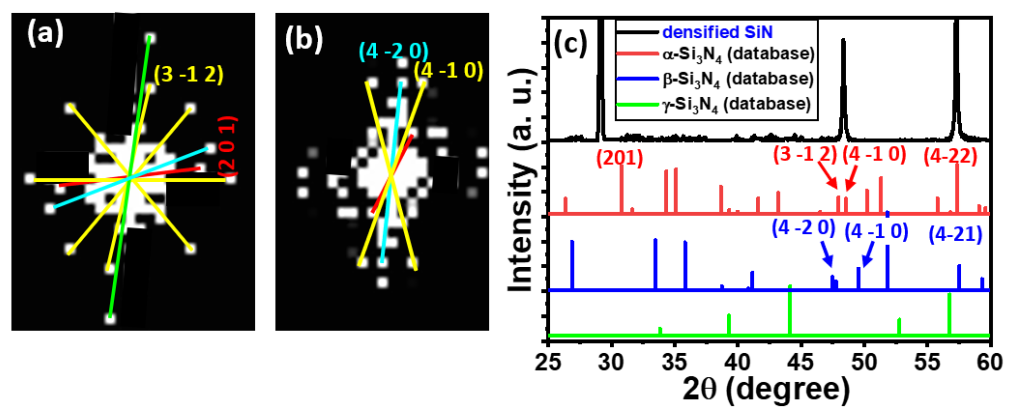

Figure 7. (a,b) SAED patterns; and (c) XRD spectra acquired for the densified, polycrystalline $\mathrm{Si}_{3} \mathrm{~N}_{4}$ generated in proximity to the Ge QDs. The appearance of clear electron diffraction spots and XRD peaks is convincing evidence for the phase transition of the $\mathrm{Si}_{3} \mathrm{~N}_{4}$ from the amorphous to the densified polycrystalline state.

\subsection{Process-Controlled Placement of Spherical-Shaped Ge QDs at Designated Spatial Locations}

Placement of our Ge QDs by design is facilitated via controlled heterogeneous nucleation and growth within lithographically patterned structures. Pattern-dependent oxidation and Ostwald ripening-based migration behavior offer additional mechanisms for controlling the QD locations. Our extensive experimental observations show that segregated Ge nuclei tend to form at the sidewall edges and near the included-angle locations of the $\mathrm{Si}_{3} \mathrm{~N}_{4}$ /c-Si ridges of asterisk-shaped configurations. These locations are also where large geometric curvatures and higher film stress occur. The preferential formation of Ge QDs at the highly stressed ridge sidewall edges and their included-angle locations could also be due to the higher density of defects at these locations and the stress relief provided by the growing Ge QD $[47,54]$. The insertion of a $\mathrm{S}_{\mathrm{i} 3} \mathrm{~N}_{4}$ overlayer with controllable thickness between the poly-SiGe spacer island and the c-Si ridge provides the tunability necessary for precise Ge QD location. Not only can we direct the Ge QDs migrating towards the designated spatial locations by creating a gradient in the concentration of released $\mathrm{Si}$ interstitials in order to activate the dynamic $\mathrm{SiO}_{2}$ destruction-construction 
mechanisms ahead of the migrating Ge QD surface, but also the sacrificial consumption of the $\mathrm{Si}_{3} \mathrm{~N}_{4}$ layer prevents the c-Si ridges themselves from being consumed up during the selective oxidation process. In this way, the inter-QD coupling barriers are directly defined by the process-controllable thicknesses of the $\mathrm{Si}_{3} \mathrm{~N}_{4}$ spacer layers and widths of lithographically-defined c-Si ridges.

\subsection{Process-Controlled Size Tunability of Ge Spherical QDs for Operation in Few-Charge Regimes}

As mentioned previously, our Ge QDs are created by using the selective oxidation of SiGe spacer islands resulting in progressive segregation, condensation, and Ostwald ripening of Ge interstitials, ultimately producing spherical-shaped Ge QDs. Thus, the Ge QD diameters are, by definition, smaller than the geometric sizes of the initial poly-SiGe spacer islands. The widths and heights of the initial SiGe spacer islands are well controlled to nanometer-scale precision by adjusting the process times for deposition and etch-back, while their lengths are essentially determined by lithographic patterning. Hence, our "hybrid patterning/self-assembly" Ge QD fabrication approach allows a higher degree of controllability for producing ultrafine QDs as compared to processes using lithography alone.

Our Ostwald ripened Ge QD assumes a perfectly spherical shape as predicted by Stekolnikov and Bechstedt [55], since their unique, solid-state migration behavior mechanically decouples the QD from its surrounding matrices of $\mathrm{SiO}_{2}, \mathrm{Si}_{3} \mathrm{~N}_{4}$ or $\mathrm{Si}$. In contrast to the highly orientation-dependent energy subbands with anisotropic, hyperfine energylevel splitting for the gate-defined Ge QDs created from heterostructures of Ge/SiGe quantum-wells or nanowires [15,30,33,34], the spherical shape of our Ge QDs is desirable for quantum-electronic devices. This is because a spherical QD has a three-dimensional, radially symmetric electrostatic potential, giving rise to atomic-like discrete orbitals [26]. Similar to the case of atomic orbitals $(1 s, 2 s, 2 p, 3 s, 3 p, \ldots)$, these orbitals are also filled sequentially with large addition energies for complete filling of shells with 2, 10, 18 electrons [26]. In particular, when the sizes of spherical Ge QDs are comparable to the Bohr's radius $(\sim 24.9 \mathrm{~nm})$ or the de Broglie wavelength and smaller, the well separated energy levels in combination with large addition energies allow the QD devices to operate in the few-charge regime. The special interest in few- and even single-charge operating regimes arises from the fact that intra-QD electron-electron interaction dominates. These operating regimes make it possible to form QD-based qubits and QD-based SETs by exploiting the electron filling and spin degrees of freedom while suppressing cotunneling and thermal noise/fluctuation effects because the spin- or charge-states are energetically well-defined and separated from other states.

\subsection{Ge QD Array for Qubits and Charge Readout Sensors}

For the case of the much smaller Si-based QDs, in addition to the challenges associated with the fabrication of closely coupled QDs, another major challenge for the practical implementation of Si QD-based qubits is the reliable measurement of quantum states within these QDs that are susceptible to environmental temperatures and defects. Highprecision charge and differential current/voltage sensing devices and associated techniques are definitely required for measuring very small output current/voltage signals (on the order of sub-nA and sub-mV, respectively) for QD qubits.

An SET or SHT, comprising a single QD capacitively coupled to source/drain reservoirs and plunger-gates through confinement barriers, is the ultimate embodiment for electronic devices controlling itinerant current with single charge precision based on Coulomb blockade effects. Their extremely high sensitivity to the charge number makes QD-SETs (or SHTs) excellent readout devices for charge- and spin-qubits. Therefore, having QD-SETs (or SHTs) favorably arranged in close proximity to the QD qubits allows us to sense minute variations of local potentials induced by charge movement in between QDs.

Our proposed self-organized Ge QDs arrays with self-aligned electrodes offer configurable flexibility in constructing QD-qubits or QD-SETs, depending on the c-Si ridges 
serving as inter-QD coupling barriers or simply acting as reservoirs. Using our proposed fabrication processes for self-aligned external electrodes of plunger gates, barrier gates, and reservoirs (Figure 2), each QD within the array is individually addressable by four self-aligned electrodes, that is, two poly-Si (or polycide) barrier gates (or one barrier gate and one reservoir), one poly-Si (or polycide) plunger gate, and a common poly-Si (or polycide) layer located in the center of the array. The barrier gates capacitively adjust inter-QD interactions within the coupling barriers of $\mathrm{Si}_{3} \mathrm{~N}_{4}$ spacer/c-Si ridge through a thin $\mathrm{SiO}_{2}$ layer, whereas the QD potential itself could be independently adjusted by means of the plunger gates or by the common electrode in the center of the array coupled by the newly-grown cladding layers of $\mathrm{SiO}_{2}$. For our demonstrated OQD array arrangements, possible QD device configurations are proposed in Figure 8. Figure 8a is a suggested coupled QDs configuration for a qubit including DQDs, one barrier gate over the coupling barrier of $\mathrm{Si}_{3} \mathrm{~N}_{4}$ spacer/c-Si fanout-ridge, two plunger gates over the cladding oxide/Ge QDs, and two source/drain reservoirs. A QD-SET configuration comprising a single Ge $\mathrm{QD}, \mathrm{Si}_{3} \mathrm{~N}_{4}$ spacer layers as tunnel barriers, c-Si ridges as source/drain reservoirs, and a poly-Si plunger gate is proposed in Figure $8 \mathrm{~b}$. Another possible configuration for a SET-inverter shown in Figure 8c comprises two QD-SETs connected in series by sharing the same c-Si ridge as reservoirs and modulated by the common poly-Si plunger gate at the center of the array. Figure $8 \mathrm{~d}$ is a proposed configuration of six QDs in a circular-ring arrangement and closely-integrated with two SETs located at the left and right terminals for proximal charge-sensing.

(a)

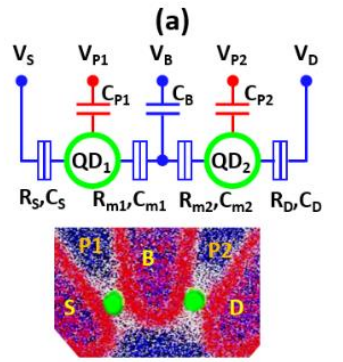

(b)
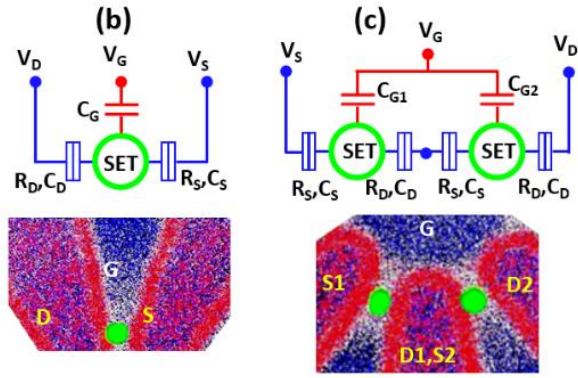

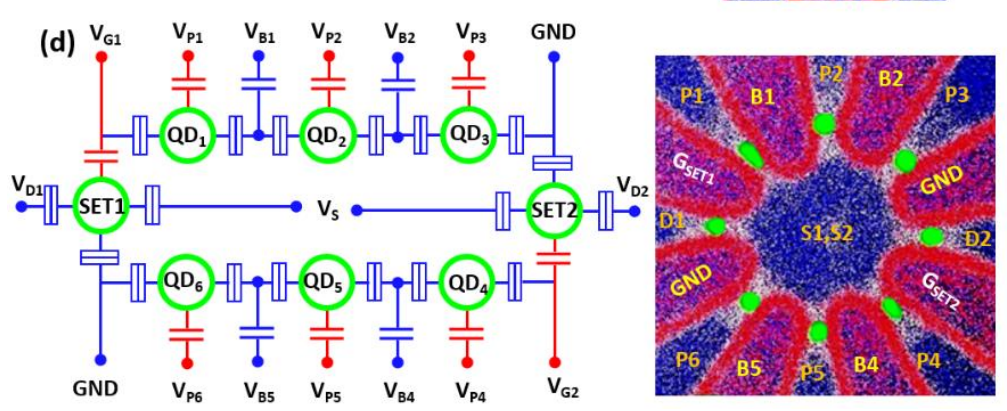

Figure 8. Possible configurations and layouts of Ge QD quantum electronic devices. (a) coupled DQDs as a qubit; (b) a QD-SET; (c) a SET logic inverter comprising two QD-SETs connected in series; and (d) a chain of QD-qubits integrated with two charge sensors of SETs.

\subsection{Proof-of-Principle Ge-QD Single-Hole Transistors Operation}

Based on our proposed self-organized heterostructures (Figure $8 b$ ) of c-Si (source) $/ \mathrm{Si}_{3} \mathrm{~N}_{4} /$ $\mathrm{Ge}-\mathrm{QD} / \mathrm{Si}_{3} \mathrm{~N}_{4} / \mathrm{c}-\mathrm{Si}$ (drain) in combination with self-aligned poly-Si electrodes (plunger gates), we have fabricated and demonstrated Ge-QD SHTs operation at $77 \mathrm{~K}$. Figure 9 shows experimental characteristics of $I_{D}-V_{D}-V_{G}$ curves (Figure $9 a$ ) and Coulomb stability diagram (Figure $9 \mathrm{~b}$ ) of $G_{D}$ contour plot measured at temperature of $77 \mathrm{~K}$. Clear oscillatory current behaviors and well-sealed Coulomb diamonds are testament to the proof-of-principle Ge-QD electronic devices operation. Each oscillatory current peak corresponds to a change of one additional hole within the Ge QDs as a result of strong Coulomb blockade effect. Each node between Coulomb diamonds represents one additional hole tunneling through 
one-particle energy levels or overcoming particle Coulomb interactions. Estimated single addition energy for holes through the Ge QDs are larger than $25 \mathrm{meV}$ from the slopes and voltage periodicity of the corresponds diamonds in Figure $9 \mathrm{~b}$.

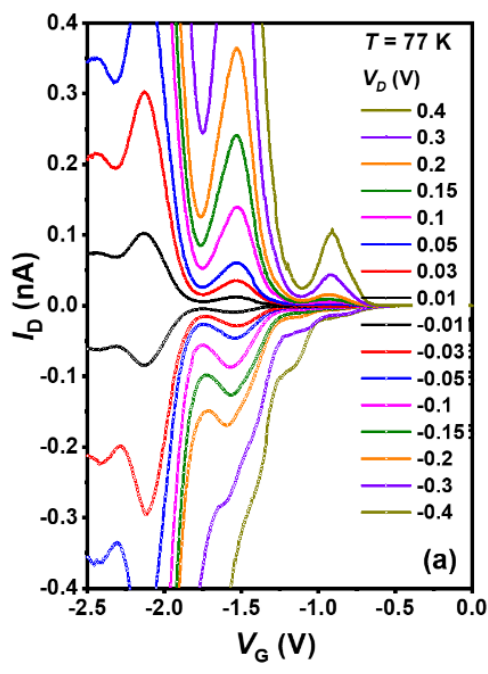

Figure 9. (a) $\mathrm{I}_{\mathrm{D}}-\mathrm{V}_{\mathrm{D}}-\mathrm{V}_{\mathrm{G}}$ curves and (b) Coulomb stability diagram of Ge-QD SHTs measured at $\mathrm{T}=77 \mathrm{~K}$.

\section{Conclusions}

An ingenious combination of lithography and self-assembled growth has allowed us to have accurate control over the placement, shapes and sizes of our "designer" Ge QDs. One novel implementation is the fabrication of closely coupled Ge QDs at designated included-angle locations of specially designed c-Si fanout-ridges providing a common platform for creating diverse QD-based quantum-electronic devices. The appeal of our Ge QD fabrication approach lies in the engineering advantages of positioning the desired number of size-tunable spherical Ge QDs at designated locations. These size-tunable Ge QDs not only share an inter-QD coupling barrier of $\mathrm{Si}_{3} \mathrm{~N}_{4}$ spacer layers/c-Si ridges in a self-organized manner, but are also electrically addressable by self-aligned electrodes. We have successfully demonstrated controllable coupling barriers of $\mathrm{Si}_{3} \mathrm{~N}_{4}$ spacers/c-Si ridges and tunneling barriers of thermally grown $\mathrm{SiO}_{2}$, respectively. All Ge QDs within our designer QD arrays are flexible for the configuration design and fabrication of qubits or readout SETs as desired, depending on the c-Si ridges serving as inter-QD coupling barriers or simply acting as reservoirs. Our proposed Ge-QD array approach offers, for the first time, a multi-dimensional parameter space for engineering novel QD electronic devices and optimizing their performance.

Author Contributions: Conceptualization, P.-W.L. and I.-H.W.; Methodology, I.-H.W. and P.-Y.H.; Validation, P.-Y.H., H.-C.L. and K.-P.P.; Formal Analysis, K.-P.P., H.-C.L. and T.G.; Data Curation, P.-W.L. and I.-H.W.; Writing - Original Draft Preparation, P.-W.L. and I.-H.W.; Writing - Review \& Editing, T.G. and P.-W.L.; Supervision, P.-W.L.; Funding Acquisition, P.-W.L. All authors have read and agreed to the published version of the manuscript.

Funding: This work was supported by the Ministry of Science and Technology, Republic of China (MOST 109-2221-E-009-022-MY3 and 108-2221-E-009-007-MY3).

Institutional Review Board Statement: Not applicable.

Informed Consent Statement: Not applicable.

Data Availability Statement: The data presented in this study are available on request from the corresponding author.

Conflicts of Interest: The authors declare no conflict of interest. 


\section{Nomenclature}

\begin{tabular}{ll}
\hline Abbreviations & Full names \\
\hline BG & barrier gate \\
CMOS & complementary metal-oxide-semiconductor \\
CB & coupling barrier \\
DQDs & double quantum dots \\
EBL & electron-beam lithography \\
EELS & electron energy loss spectroscopy \\
EDS & energy dispersive x-ray spectroscopy \\
Dit & interface trap density \\
LPCVD & low-pressure chemical vapor deposition \\
OQDs & octuple QDs \\
PL & photoluminescence \\
PG & plunger gate \\
QQDs & quadruple QDs \\
qubit & quantum bit \\
QD & quantum dot \\
SEM & scanning electron microscopy (SEM) \\
STEM & scanning transmission electron microscopy \\
SAED & selected area electron diffraction \\
SOI & silicon-on-insulator \\
SET & single-electron transistor \\
SHT & single-hole transistor \\
TEM & transmission electron microscopy \\
TQDs & triple quantum dots \\
2DEG & two-dimensional electron gas \\
2DHG & two-dimensional hole gas \\
VLSI & very large scale integrated circuits \\
XRD & X-ray diffraction \\
\hline &
\end{tabular}

\section{References}

1. Mi, X.; Benito, M.; Putz, S.; Zajac, D.M.; Taylor, J.M.; Burkard, G.; Petta, J.R. A coherent spin-photon interface in silicon. Nature 2018, 555, 599-603. [CrossRef]

2. Mehta, K.K.; Bruzewicz, C.D.; McConnell, R.; Ram, R.J.; Sage, J.M.; Chiaverini, J. Integrated optical addressing of an ion qubit. Nat. Nanotechnol. 2016, 11, 1066-1070. [CrossRef]

3. Devoret, M.H.; Schoelkopf, R.J. Superconducting Circuits for Quantum Information: An Outlook. Science 2013, 339, 1169-1174. [CrossRef]

4. London, P.; Scheuer, J.; Cai, J.; Schwarz, I.; Retzker, A.; Plenio, M.B.; Katagiri, M.; Teraji, T.; Koizumi, S.; Isoya, J.; et al. Detecting and Polarizing Nuclear Spins with Double Resonance on a Single Electron Spin. Phys. Rev. Lett. 2013, 111, 067601. [CrossRef]

5. Li, H.-O.; Cao, G.; Yu, G.-D.; Xiao, M.; Guo, G.-C.; Jiang, H.-W.; Guo, G.-P. Conditional rotation of two strongly coupled semiconductor charge qubits. Nat. Commun. 2015, 6, 7681. [CrossRef]

6. Samkharadze, N.; Zheng, G.; Kalhor, N.; Brousse, D.; Sammak, A.; Mendes, U.C.; Blais, A.; Scappucci, G.; Vandersypen, L.M.K. Strong spin-photon coupling in silicon. Science 2018, 359, 1123-1127. [CrossRef]

7. Tosi, G.; Mohiyaddin, F.A.; Schmitt, V.; Tenberg, S.; Rahman, R.; Klimeck, G.; Morello, A. Silicon quantum processor with robust long-distance qubit couplings. Nat. Commun. 2017, 8, 1-11. [CrossRef]

8. Pillarisetty, R.; Thomas, N.; George, H.; Singh, K.; Roberts, J.; Lampert, L.; Amin, P.; Watson, T.; Zheng, G.; Torres, J.; et al. In Proceedings of the Qubit Device Integration Using Advanced Semiconductor Manufacturing Process Technology, San Francisco, CA, USA, 1-5 December 2018. [CrossRef]

9. Veldhorst, M.; Eenink, H.G.J.; Yang, H.; Dzurak, A.S. Silicon CMOS architecture for a spin-based quantum computer. Nat. Commun. 2017, 8, 1-8. [CrossRef]

10. Yoneda, J.; Takeda, K.; Otsuka, T.; Nakajima, T.; Delbecq, M.; Allison, G.; Honda, T.; Kodera, T.; Oda, S.; Hoshi, Y.; et al. A quantum-dot spin qubit with coherence limited by charge noise and fidelity higher than $99.9 \%$. Nat. Nanotechnol. 2017, 13, 102-106. [CrossRef]

11. Watson, T.F.; Philips, S.G.J.; Kawakami, E.; Ward, D.; Scarlino, P.; Veldhorst, M.; Savage, D.; Lagally, M.G.; Friesen, M.; Coppersmith, S.; et al. A programmable two-qubit quantum processor in silicon. Nature 2018, 555, 633-637. [CrossRef]

12. Maurand, R.; Jehl, X.; Kotekar-Patil, D.; Corna, A.; Bohuslavskyi, H.; Laviéville, R.; Hutin, L.; Barraud, S.; Vinet, M.; Sanquer, M.; et al. A CMOS silicon spin qubit. Nat. Commun. 2016, 7, 13575. [CrossRef] [PubMed] 
13. Meunier, T.; Hutin, L.; Bertrand, B.; Thonnart, Y.; Pillonnet, G.; Billiot, G.; Jacquinot, H.; Casse, M.; Barraud, S.; Kim, Y.-J.; et al. In Proceedings of the Towards Scalable Quantum Computing based on Silicon Spin, Kyoto, Japan, 9-14 June 2019. [CrossRef]

14. Pillarisetty, R.; George, H.C.; Watson, T.F.; Lampert, L.; Thomas, N.; Bojarski, S.; Amin, P.; Caudillo, R.; Henry, E.; Kashani, N.; et al. High volume electrical characterization of semiconductor qubits. In Proceedings of the 2019 IEEE International Electron Devices Meeting (IEDM), San Francisco, CA, USA, 7-11 December 2019; pp. 737-740.

15. Masruroh, H.; Diniaty, A. Development of Chem in Action instructional media based on drill and practice in chemical equilibrium material for students in senior high school. AIP Conf. Proc. 2020, 2229, 020005. [CrossRef]

16. Yang, C.H.; Leon, R.C.C.; Hwang, J.C.C.; Saraiva, A.; Tanttu, T.; Huang, W.; Lemyre, J.C.; Chan, K.W.; Tan, K.Y.; Hudson, F.E.; et al. Operation of a silicon quantum processor unit cell above one kelvin. Nature 2020, 580, 350-354. [CrossRef] [PubMed]

17. Zwanenburg, F.A.; Dzurak, A.; Morello, A.; Simmons, M.; Hollenberg, L.; Klimeck, G.; Rogge, S.; Coppersmith, S.; Eriksson, M.A. Silicon quantum electronics. Rev. Mod. Phys. 2013, 85, 961-1019. [CrossRef]

18. Angus, S.J.; Ferguson, A.J.; Dzurak, A.S.; Clark, R.G. Gate-Defined Quantum Dots in Intrinsic Silicon. Nano Lett. 2007, 7, 2051-2055. [CrossRef] [PubMed]

19. Klos, J.; Sun, B.; Beyer, J.; Kindel, S.; Hellmich, L.; Knoch, J.; Schreiber, L.R. Spin Qubits Confined to a Silicon Nano-Ridge. Appl. Sci. 2019, 9, 3823. [CrossRef]

20. Zajac, D.M.; Hazard, T.M.; Mi, X.; Wang, K.; Petta, J.R. A reconfigurable gate architecture for Si/SiGe quantum dots. Appl. Phys. Lett. 2015, 106, 223507. [CrossRef]

21. Andrews, R.W.; Jones, C.; Reed, M.D.; Jones, A.M.; Ha, S.D.; Jura, M.P.; Kerckhoff, J.; Levendorf, M.; Meenehan, S.; Merkel, S.T.; et al. Quantifying error and leakage in an encoded Si/SiGe triple-dot qubit. Nat. Nanotechnol. 2019, 14, 747-750. [CrossRef] [PubMed]

22. Takakura, T.; Noiri, A.; Obata, T.; Otsuka, T.; Yoneda, J.; Yoshida, K.; Tarucha, S. Single to quadruple quantum dots with tunable tunnel couplings. Appl. Phys. Lett. 2014, 104, 113109. [CrossRef]

23. Volk, C.; Zwerver, A.M.J.; Mukhopadhyay, U.; Eendebak, P.T.; Van Diepen, C.J.; Dehollain, J.P.; Hensgens, T.; Fujita, T.; Reichl, C.; Wegscheider, W.; et al. Loading a quantum-dot based “Qubyte" register. npj Quantum Inf. 2019, 5, 29. [CrossRef]

24. Thorbeck, T.; Zimmerman, N.M. Formation of strain-induced quantum dots in gated semiconductor nanostructures. AIP Adv. 2015, 5, 087107. [CrossRef]

25. Brauns, M.; Amitonov, S.V.; Spruijtenburg, P.-C.; Zwanenburg, F.A. Palladium gates for reproducible quantum dots in silicon. Sci. Rep. 2018, 8, 1-8. [CrossRef] [PubMed]

26. Tarucha, S.; Austing, D.G.; Honda, T.; van der Hage, R.J.; Kouwenhoven, L.P. Shell Filling and Spin Effects in a Few Electron Quantum Dot. Phys. Rev. Lett. 1996, 77, 3613-3616. [CrossRef] [PubMed]

27. Horibe, K.; Kodera, T.; Oda, S. Lithographically defined few-electron silicon quantum dots based on a silicon-on-insulator substrate. Appl. Phys. Lett. 2015, 106, 083111. [CrossRef]

28. Mizokuchi, R.; Oda, S.; Kodera, T. Physically defined triple quantum dot systems in silicon on insulator. Appl. Phys. Lett. 2019, 114, 073104. [CrossRef]

29. Zhou, Y.; Han, W.; Chang, L.-T.; Xiu, F.; Wang, M.; Oehme, M.; Fischer, I.A.; Schulze, J.; Kawakami, R.K.; Wang, K.L. Electrical spin injection and transport in germanium. Phys. Rev. B 2011, 84, 125323. [CrossRef]

30. Hendrickx, N.; Franke, D.P.; Sammak, A.; Scappucci, G.; Veldhorst, M. Fast two-qubit logic with holes in germanium. Nature 2020, 577, 487-491. [CrossRef]

31. Froning, F.N.M.; Rehmann, M.K.; Ridderbos, J.; Brauns, M.; Zwanenburg, F.; Li, A.; Bakkers, E.P.A.M.; Zumbühl, D.M.; Braakman, F.R. Single, double, and triple quantum dots in Ge/Si nanowires. Appl. Phys. Lett. 2018, 113, 073102. [CrossRef]

32. 32. Vukusic, L.; Kukucka, J.; Watzinger, H.; Katsaros, G. Fast hole tunneling times in germanium hut wires probed by single-shot reflectometry. Nano Lett. 2017, 17, 5706. [CrossRef]

33. Scappucci, G.; Kloeffel, C.; Zwanenburg, F.A.; Loss, D.; Myronov, M.; Zhang, J.-J.; De Franceschi, S.; Katsaros, G.; Veldhorst, M. The germanium quantum information route. Nat. Rev. Mater. 2020, 6, 926-943. [CrossRef]

34. Hendrickx, N.W.; Veldhorst, M. An array of four germanium qubits. Nature 2021. [CrossRef]

35. Kuo, M.H.; Wang, C.C.; Lai, W.T.; George, T.; Li, P.W. Designer Ge quantum dots on Si: A heterostructure configuration with enhanced optoelectronic performance. Appl. Phys. Lett. 2012, 101, 223107. [CrossRef]

36. Liao, P.H.; Kuo, M.H.; Tien, C.W.; Chang, Y.L.; Hong, P.Y.; George, T.; Lin, H.C.; Li, P.W. In Proceedings of the Self-Organized Gate Stack of Ge Nanosphere $/ \mathrm{SiO}_{2} / \mathrm{Si1}-x \mathrm{Gex}$ Enables Ge-Based Monolithically-Integrated Electronics and Photonics on Si Platform, Honolulu, HI, USA, 18-22 June 2018; pp. 157-158. [CrossRef]

37. Huang, T.-L.; Peng, K.-P.; Chen, C.-L.; Lin, H.-C.; George, T.; Li, P.-W. Tunable diameter and spacing of double Ge quantum dots using highly-controllable spacers and selective oxidation of SiGe. Sci. Rep. 2019, 9, 1-8. [CrossRef]

38. Peng, K.-P.; Chen, C.-L.; Tang, Y.-T.; Kuo, D.; George, T.; Lin, H.-C.; Li, P.-W. In Proceedings of the Self-Organized Pairs of Ge Double Quantum Dots with Tunable Sizes and Spacings Enable Room-Temperature Operation of Qubit and Single-Electron Devices, San Francisco, CA, USA, 7-11 December 2019. [CrossRef]

39. Chen, K.-H.; Wang, C.-C.; George, T.; Li, P.-W. The role of Si interstitials in the migration and growth of Ge nanocrystallites under thermal annealing in an oxidizing ambient. Nanoscale Res. Lett. 2014, 9, 339. [CrossRef]

40. George, T.; Li, P.W.; Chen, K.H.; Peng, K.P.; Lai, W.T. 'Symbiotic' semiconductors: Unusual and counter-intuitive Ge/Si/O interactions. J. Phys. D: Appl. Phys. 2017, 50, 105101. [CrossRef] 
41. Chen, K.H.; Wang, C.C.; George, T.; Li, P.W. The pivotal role of SiO formation in the migration and Ostwald ripening of Ge quantum dots. Appl. Phys. Lett. 2014, 105, 122102. [CrossRef]

42. Chen, G.-L.; Kuo, D.M.T.; Lai, W.-T.; Li, P.-W. Tunneling spectroscopy of a germanium quantum dot in single-hole transistors with self-aligned electrodes. Nanotechnology 2007, 18. [CrossRef]

43. Chen, I.-H.; Chen, K.-H.; Lai, W.-T.; Li, P.-W. Single Germanium Quantum-dot Placement Along With Self-Aligned Electrodes for Effective Management of Single Charge Tunneling. IEEE Trans. Electron Devices 2012, 59, 3224-3230. [CrossRef]

44. Chen, I.H.; Lai, W.T.; Li, P.W. Realization of solid-state nanothermometer using Ge quantum-dot single-hole transistor in few-hole regime. Appl. Phys. Lett. 2014, 104, 243506. [CrossRef]

45. Chen, K.-H.; Chien, C.-Y.; Li, P.-W. Precise Ge quantum dot placement for quantum tunneling devices. Nanotechnology 2009, 21, 055302. [CrossRef] [PubMed]

46. Liao, P.H.; Hsu, T.C.; Chen, K.H.; Cheng, T.H.; Hsu, T.M.; Wang, C.C.; George, T.; Li, P.W. Size-tunable strain engineering in Ge nanocrystals embedded within $\mathrm{SiO}_{2}$ and $\mathrm{Si}_{3} \mathrm{~N}_{4}$. Appl. Phys. Letts. 2014, 105, 172106. [CrossRef]

47. Kuo, Y.-H.; Chiu, S.-H.; Tien, C.-W.; Lin, S.-D.; Chang, W.-H.; George, T.; Lin, H.-C.; Li, P.-W. Nitride-stressor and quantum-size engineering in Ge quantum-dot photoluminescence wavelength and exciton lifetime. Nano Futur. 2020, 4, 015001. [CrossRef]

48. Kuo, M.H.; Chou, S.K.; Pan, Y.W.; Lin, S.D.; George, T.; Li, P.W. “Embedded Emitters": Direct bandgap Ge nanodots within SiO 2 . J. Appl. Phys. 2016, 120, 233106. [CrossRef]

49. Peng, K.-P.; Kuo, Y.-H.; Chang, L.-H.; Hsiao, C.-N.; Chung, T.-F.; George, T.; Lin, H.-C.; Li, P.-W. Silicon nitride engineering: Role of hydrogen-bonding in Ge quantum dot formation. Semicond. Sci. Technol. 2020, 35, 105018. [CrossRef]

50. Gurvich, L.V.; Iorish, V.S.; Yungman, V.S.; Dorofeeva, O.V. Thermodynamic Properties as a Function of Temperature. In CRC Handbook of Chemistry and Physics, 84th ed.; Lide, D.R., Ed.; CRC Press: Boca Raton, FL, USA, 2003; pp. 575-584.

51. Chien, C.Y.; Chang, Y.J.; Chen, K.H.; Lai, W.T.; George, T.; Scherer, A.; Li, P.W. Nanoscale, catalytically enhanced local oxidation of silicon-containing layers by 'burrowing' Ge quantum dots. Nanotechnology 2011, 22, 435602. [CrossRef] [PubMed]

52. Peng, K.-P.; Huang, T.-L.; George, T.; Lin, H.-C.; Li, P.-W.; George, T. Ge nanodot-mediated densification and crystallization of low-pressure chemical vapor deposited Si3N4 for advanced complementary metal-oxide-semiconductor photonics and electronics applications. Nanotechnology 2019, 30, 405201. [CrossRef] [PubMed]

53. Lai, W.T.; Yang, K.C.; Liao, P.H.; George, T.; Li, P.W. Gate-stack engineering for self-organized Ge-dot $/ \mathrm{SiO}_{2} / \mathrm{SiGe-shell} \mathrm{MOS}$ capacitors. Frontiers in Materials 2016, 3, 00005. [CrossRef]

54. Chen, H.-Y.; Peng, K.-P.; George, T.; Lin, H.-C.; Li, P.-W. Coordinated and Simultaneous Formation of Paired Ge Quantum Dots by Thermal Oxidation of Designer Poly-SiGe Spacer Structures. IEEE Trans. Nanotechnol. 2020, 19, 436-438. [CrossRef]

55. Stekolnikov, A.A.; Bechstedt, F. Shape of free and constrained group-IV crystallites: Influence of surface energies. Phys. Rev. $B$ 2005, 72, 125326. [CrossRef] 